\title{
SIMPLE SEMIGROUP GRADED RINGS
}

\author{
PATRIK NYSTEDT \\ University West, Department of Engineering Science, SE-46186 Trollhättan, Sweden \\ JOHAN ÖINERT \\ Centre for Mathematical Sciences, P.O. Box 118, Lund University, SE-22100 Lund, \\ Sweden
}

\begin{abstract}
We show that if $R$ is a, not necessarily unital, ring graded by a semigroup $G$ equipped with an idempotent $e$ such that $G$ is cancellative at $e$, the non-zero elements of $e G e$ form a hypercentral group and $R_{e}$ has a non-zero idempotent $f$, then $R$ is simple if and only if it is graded simple and the center of the corner subring $f R_{e G e} f$ is a field. This is a generalization of a result of E. Jespers' on the simplicity of a unital ring graded by a hypercentral group. We apply our result to partial skew group rings and obtain necessary and sufficient conditions for the simplicity of a, not necessarily unital, partial skew group ring by a hypercentral group. Thereby, we generalize a very recent result of $\mathrm{D}$. Gonçalves'. We also point out how E. Jespers' result immediately implies a generalization of a simplicity result, recently obtained by A. Baraviera, W. Cortes and M. Soares, for crossed products by twisted partial actions.
\end{abstract}

\section{INTRODUCTION}

Suppose that $R$ is an associative, not necessarily unital, ring and $G$ is a semigroup, i.e. a non-empty set equipped with an associative binary operation $G \times G \ni(g, h) \mapsto g h \in G$. Recall that $R$ is called $G$-graded if there for each $g \in G$ is an additive subgroup $R_{g}$ of $R$ such that $R=\oplus_{g \in G} R_{g}$ and the inclusion $R_{g} R_{h} \subseteq R_{g h}$ holds for all $g, h \in G$.

The investigation of semigroup graded rings has been carried out by many authors, see e.g. [1, 6, 17, 8, 9, 11, 17, 20, 23, 27, 30, 34, 35, 37. For an excellent overview of the theory of semigroup graded rings, we refer the reader to A. V. Kelarev's extensive book [26], and the references therein.

Since many ring constructions are special cases of semigroup graded rings, e.g. monomial rings, crossed products, skew polynomial rings, twisted semigroup rings, skew power series rings, edge and path algebras, generalized matrix rings, incidence algebras and category

E-mail addresses: Patrik.Nystedt@hv.se, Johan.Oinert@math.lth.se.

2010 Mathematics Subject Classification. 16W50, 16D25, 16U70, 16S35.

Key words and phrases. semigroup graded ring, partial skew group ring, simplicity. 
graded rings, the theory of semigroup graded rings can be applied to the study of other less general constructions, giving new results for several constructions simultaneously, and unifying theorems obtained earlier.

An important problem in the investigation of semigroup graded rings is to explore how properties of the whole ring $R$ are connected to properties of subrings $R_{H}=\bigoplus_{g \in H} R_{g}$ where $H$ runs over subsemigroups of $G$. Many results of this sort are known for finiteness conditions, nil and radical properties, semisimplicity, semiprimeness and semiprimitivity (see the references in [24] and [25]).

The aim of this article is to establish a similar result (see Theorem 2) for simple semigroup graded rings. Recall that an (two-sided) ideal $I$ of a $G$-graded ring $R$ is said to be a graded ideal (or G-graded ideal) if $I=\oplus_{g \in G}\left(I \cap R_{g}\right)$ holds. A $G$-graded ring $R$ is called graded simple (or $G$-graded simple) if $R$ and $\{0\}$ are its only graded ideals. Clearly, graded simplicity is a necessary condition for simplicity. In the case when $G$ is a hypercentral group, that is when every non-trivial factor group of $G$ has non-trivial center, E. Jespers [22] has determined precisely when unital $G$-graded rings are simple.

Theorem 1 (E. Jespers [22]). If $G$ is a hypercentral group and $R$ is a G-graded unital ring, then $R$ is simple if and only if $R$ is graded simple and the center of $R$ is a field.

Suppose that $R$ is a ring graded by a semigroup equipped with a zero element, i.e. an element $\theta \in G$ satisfying $\theta g=g \theta=\theta$, for $g \in G$. This implies that whenever $R_{\theta}$ is non-zero and there is a non-zero $g \in G$ such that $R_{g}$ is non-zero, $R_{\theta}$ is a non-trivial ideal of $R$ and, hence, $R$ is not simple. We therefore, throughout this article, make the assumption that if $G$ has a zero element $\theta$, then $R_{\theta}=\{0\}$. To state our generalization of Theorem 1, we need to introduce the following semigroup notion. If $G$ is a semigroup and $e$ is a non-zero idempotent in $G$, then we say that $G$ is cancellative at $e$ if for any $a, b, x, y \in G$, a relation of the form eaxbe $=$ eaybe $\neq \theta$, implies that $x=y$. Clearly, all groups are cancellative at identity elements. Also, all semigroups induced by groupoids are cancellative at identity elements (see Remark 10).

Theorem 2. If $R$ is a ring graded by a semigroup $G$ equipped with a non-zero idempotent $e$ such that $G$ is cancellative at $e$, the non-zero elements of eGe form a hypercentral group and $R_{e}$ has a non-zero idempotent $f$, then $R$ is simple if and only if it is graded simple and the center of the corner subring $f R_{e G e} f$ is a field.

Here we would like to make two remarks. First of all, note that the above result might at first glance seem more general than it actually is. In fact, graded simplicity of $R$ often forces the semigroup $G$ to be an inverse semigroup (see Remark 9). Secondly, up until recently, the authors of the present article (and presumably also the authors of e.g. 44, [10]) were unaware of the existence of E. Jespers' simplicity results in [21] and [22], as well as A. D. Bell's in [5]. The technique used to prove the simplicity criterion for skew group rings in [33] is slightly different from the one used in [21] and [22], but can in fact, after minor adjustments, be used to give a more direct proof of the main result of [21].

This article is organized as follows. 
In Section 2, we recall the relevant definitions concerning semigroup graded rings and prove Theorem 2 .

In Section 3, we apply Theorem 2 to partial skew group rings. Partial group actions were introduced by R. Exel in the context of crossed product $\mathrm{C}^{*}$-algebras [14]. A decade later, the investigation of its algebraic counterpart (the partial skew group rings) began by [13]. We obtain necessary and sufficient conditions for the simplicity of, not necessarily unital, partial skew group rings by hypercentral groups. Thereby, we generalize a very recent result of D. Gonçalves' [18]. At the end of the section, we also point out how E. Jespers' result immediately implies a generalization of a simplicity result, recently obtained by $\mathrm{A}$. Baraviera, W. Cortes and M. Soares, for crossed products by twisted partial actions.

\section{Semigroup Graded Rings}

At the end of this section, we show Theorem 2. To this end, we show a series of results concerning ideals in semigroup graded rings (see Lemma 3, Lemma 5, Proposition 66 and Proposition (7).

We begin by fixing the notation. Throughout this section, $R$ denotes a ring graded by a semigroup $G$. Take $r \in R$. There are unique $r_{g} \in R_{g}$, for $g \in G$, such that all but finitely many of them are zero and $r=\sum_{g \in G} r_{g}$. We let the support of $r$, denoted by $\operatorname{Supp}(r)$, be the set of $g \in G$ such that $r_{g} \neq 0$. The cardinality of $\operatorname{Supp}(r)$ is denoted by $|\operatorname{Supp}(r)|$. The element $r$ is called homogeneous if $|\operatorname{Supp}(r)| \leq 1$. If $r \in R_{g} \backslash\{0\}$, for some $g \in G$, then we write $\operatorname{deg}(r)=g$.

Lemma 3. Let $G$ be a group and $R$ a unital $G$-graded ring. If $R$ is graded simple and $I$ is a non-zero $G / Z(G)$-graded ideal of $R$, then for each non-zero $r \in I$, there is a non-zero $r^{\prime} \in I \cap R_{Z(G)} \cap Z(R)$ with $\left|\operatorname{Supp}\left(r^{\prime}\right)\right| \leq|\operatorname{Supp}(r)|$. If, in addition, the ring $Z(R)$ is a field, then $R$ is $G / Z(G)$-graded simple.

Proof. This is Proposition 4 of [22]. We show this result by a different method. Let $I$ be a non-zero $G / Z(G)$-graded ideal of $R$ and take a non-zero $r \in I$. Choose $g \in G$ such that $r_{g} \neq 0$. Since $R$ is graded simple, we get that $R r_{g} R=R$. In particular, we get that $1=\sum_{i=1}^{n} s_{i} r_{g} t_{i}$ for some homogeneous $s_{i}, t_{i} \in R$. Therefore, there is $j \in\{1, \ldots, n\}$ such that $s_{j} r_{g} t_{j} \neq 0$ and $\operatorname{deg}\left(s_{j} r_{g} t_{j}\right)=e$. By replacing $r$ with $s_{j} r t_{j}$ we can assume that $r_{e} \neq 0$. Since $I$ is $G / Z(G)$-graded, the $Z(G)$-degree part of $r$ belongs to $I$ and is non-zero (since $\left.r_{e} \neq 0\right)$. Therefore, we can assume that $r$ is a non-zero element belonging to $I \cap R_{Z(G)}$.

Now put $J=\left\{s_{e} \mid s \in R r R, \operatorname{Supp}(s) \subseteq \operatorname{Supp}(r)\right\}$. We want to show that $1 \in J$. Note that $J$ is a non-zero ideal of $R_{e}$ and hence that $R J R$ is a non-zero graded ideal of $R$. By graded simplicity of $R$ we get that $R J R=R$. Thus, there are $s^{(1)}, \ldots, s^{(n)} \in R r R$ and $v_{i}, w_{i} \in R$, for $i \in\{1, \ldots, n\}$, such that $1=\sum_{i=1}^{n} v_{i} s_{e}^{(i)} w_{i}$ and $\operatorname{Supp}\left(s^{(i)}\right) \subseteq \operatorname{Supp}(r)$. This implies that $\operatorname{deg}\left(v_{i}\right) \operatorname{deg}\left(w_{i}\right)=e$ for $i \in\{1, \ldots, n\}$. Put $s=\sum_{i=1}^{n} v_{i} s^{(i)} w_{i}$. Then $s \in I$ and since $\operatorname{Supp}\left(s^{(i)}\right) \subseteq \operatorname{Supp}(r) \subseteq R_{Z(G)}$, we get that $\operatorname{Supp}(s) \subseteq \cup_{i=1}^{n} \operatorname{deg}\left(v_{i}\right) \operatorname{Supp}\left(s^{(i)}\right) \operatorname{deg}\left(w_{i}\right) \subseteq$

$\cup_{i=1}^{n} \operatorname{deg}\left(v_{i}\right) \operatorname{deg}\left(w_{i}\right) \operatorname{Supp}\left(s^{(i)}\right) \subseteq \cup_{i=1}^{n} e \operatorname{Supp}(r)=\operatorname{Supp}(r)$. Therefore, $1=\sum_{i=1}^{n} v_{i} s_{e}^{(i)} w_{i}=$ $s_{e} \in J$. 
Now pick a non-zero element $r^{\prime} \in I$ with $\left|\operatorname{Supp}\left(r^{\prime}\right)\right|$ minimal. By the above, we can assume that $r_{e}^{\prime}=1$ and that $r^{\prime} \in I \cap R_{Z(G)}$. Take $g \in G$ and $t \in R_{g}$. Since $r_{e}^{\prime}=1$ and $\operatorname{Supp}\left(r^{\prime}\right) \subseteq Z(G)$, we get that $\left|\operatorname{Supp}\left(r^{\prime} t-t r^{\prime}\right)\right|<\left|\operatorname{Supp}\left(r^{\prime}\right)\right|$. By the assumptions on $r^{\prime}$ we get that $\operatorname{Supp}\left(r^{\prime} t-t r^{\prime}\right)=\emptyset$ and hence that $r^{\prime} t-t r^{\prime}=0$. Therefore $r^{\prime} \in Z(R)$.

Remark 4. Recall that if $G$ is a group with identity element $e$, then the ascending central series of $G$ is the sequence of subgroups $Z_{i}(G)$, for non-negative integers $i$, defined recursively by $Z_{0}(G)=\{e\}$ and, given $Z_{i}(G)$, for some non-negative integer $i, Z_{i+1}(G)$ is defined to be the set of $g \in G$ such that for every $h \in G$, the commutator $[g, h]=g h g^{-1} h^{-1}$ belongs to $Z_{i}(G)$. For infinite groups this process can be continued to infinite ordinal numbers by transfinite recursion. For a limit ordinal $O$, we define $Z_{O}(G)=\cup_{i<O} Z_{i}(G)$. If $G$ is hypercentral, then $Z_{O}(G)=G$ for some limit ordinal $O$. For the details concerning this construction, see [36].

Lemma 5. If $G$ is a hypercentral group and $R$ is a $G$-graded ring with the property that for each $i<O$ the ring $R$ is $G / Z_{i}(G)$-graded simple, then $R$ is simple.

Proof. Take a non-zero ideal $J$ of $R$ and a non-zero $a \in J$. We show that $\langle a\rangle=R$. Since $\cup_{i} Z_{i}(G)=G$ and $\operatorname{Supp}(a)$ is finite, we can conclude that there is some $i$ such that $\operatorname{Supp}(a) \subseteq Z_{i}(G)$. Then $\langle a\rangle$ is a non-zero $G / Z_{i}(G)$-graded ideal of $R$. Since $R$ is $G / Z_{i}(G)$ graded simple, we get that $\langle a\rangle=R$, which shows that $J=R$.

Proposition 6. If $R$ is a simple ring graded by a semigroup $G$ equipped with a non-zero idempotent $e$ and $R_{e}$ contains a non-zero idempotent $f$, then the center of the corner subring $f R_{e G e} f$ is a field.

Proof. Take a non-zero $x$ in $Z\left(f R_{e G e} f\right)$. Since the ideal $R x R$ of $R$ is non-zero and $R$ is simple, we get that $R x R=R$. In particular, $f$ equals a finite sum of elements of the form $y_{i} x z_{i}$, for $i \in\{1, \ldots, n\}$, where each $y_{i}$ and each $z_{i}$ is homogeneous. Hence $f=f \cdot f \cdot f=\sum_{i=1}^{n} f y_{i} f x f z_{i} f$ so we may assume that $x_{i}, y_{i} \in f R_{e G e} f$ for all $i$. But since $x$ belongs to $Z\left(f R_{e G e} f\right)$ we get that $f=w x=x w$ for some $w \in f R_{e G e} f$. All that is left to show now is that $w \in Z\left(f R_{e G e} f\right)$. Take $v \in f R_{e G e} f$. Then, since $x$ commutes with $v$, we get that $w v=w v f=w v x w=w x v w=f v w=v w$.

Proposition 7. Suppose that $G$ is a semigroup and $R$ is a ring graded by $G$. If $R$ is graded simple and there is a non-zero idempotent $e \in G$ such that $G$ is cancellative at $e$, the nonzero elements of eGe form a hypercentral group, $R_{e}$ contains a non-zero idempotent $f$ and the center of the corner subring $f R_{e G e} f$ is a field, then $R$ is simple.

Proof. Let $H$ denote the group of non-zero elements of $e G e$ and put $S=f R_{H} f$. We claim that $S$ is simple. Assume for a moment that the claim holds. We show that $R$ is simple. Take a non-zero ideal $I$ of $R$ and a non-zero $x \in I$. Take $g \in G$ such that $x_{g} \neq 0$. Let $J$ denote the smallest two-sided ideal of $R$ containing $x_{g}$, i.e. $J=\mathbb{Z} x_{g}+R x_{g}+x_{g} R+R x_{g} R$. Since $J$ is a graded ideal and $R$ is graded simple, we get that $f \in J$. Since $f=f^{3}$, this implies that $f \in f J f$ and thus we can write $f=\sum_{i=1}^{n} f y_{i} x_{g} z_{i} f$ for some homogeneous $y_{i}, z_{i} \in R$. From the fact that $f$ is non-zero it now follows that there is $j \in\{1, \ldots, n\}$ such that $f y_{j} x_{g} z_{j} f$ is non-zero. Now put $x^{\prime}=f y_{j} x z_{j} f$. By the construction of $x^{\prime}$ it follows that 
$x^{\prime} \in I \cap S$. Since $G$ is cancellative at $e$ it also follows that $x^{\prime}$ is non-zero. We thus get that $I \cap S \neq\{0\}$. But since $S$ is simple, we get that $I \cap S=S$, or, equivalently, that $S \subseteq I$. This implies, in particular, that $f \in I$. Since $f$ is homogeneous and $R$ is graded simple, we thus get that $I \supseteq R f R=R$. Hence $I=R$.

Now we show the claim in the beginning of the proof, i.e. that $S$ is simple. Let $Z_{i}(H)$, for $i \geq 0$, be the ascending central series of $H$ (see Remark 4 ). By induction over $i$ we now show that for each $i \in I$, the ring $S$ is $H / Z_{i}(H)$-graded simple.

First we show the base case: $i=0$. Since $H / Z_{0}(H)=H /\{e\}=H$, we need to show that $S$ is $H$-graded simple. Suppose that $J$ is a non-zero $H$-graded ideal of $S$. Then $R J R$ is a non-zero $G$-graded ideal of $R$. Since $R$ is graded simple, we get that $R J R=R$. In particular, we get that $f \in R J R$ and hence $f=\sum_{i=1}^{n} y_{i} x_{i} z_{i}$ for some $x_{i} \in J$ and homogeneous $y_{i}, z_{i} \in R$. From this we get that $f=f^{3}=\sum_{i=1}^{n} f y_{i} f x_{i} f z_{i} f \in S J S \subseteq J$. Since $f$ is a multiplicative identity of $S$, we get that $J=S$.

Now we show the induction step. Suppose that the statement is true for some $i$, i.e. that $S$ is $H / Z_{i}(H)$-graded simple. By Lemma 3. we get that $S$ is $\frac{H / Z_{i}(H)}{Z\left(H / Z_{i}(H)\right)}$-graded simple. Since the center of $H / Z_{i}(H)$ equals $Z_{i+1}(H) / Z_{i}(H)$ we get that $S$ is $\frac{H / Z_{i}(H)}{Z_{i+1}(H) / Z_{i}(H)}$-graded simple, i.e. that $S$ is $H / Z_{i+1}(H)$-graded simple and the induction step is complete.

By Lemma 5, we get that $S$ is simple.

Proof of Theorem 2. This follows immediately from Propositions 6 and 7.

Remark 8. Theorem 1 (and hence Theorem 2) can not be generalized to arbitrary groups (or semigroups). Indeed, let $G$ denote the free group on two generators, let $K$ be a field and suppose that $R=K[G]$ denotes the group ring of $G$ over $K$. Since $G$ is an ICC-group, i.e. each non-identity element has an infinite conjugacy class, it follows from [33, Proposition 5.3] that $Z(R)=K$ is a field. It is not difficult to see that $R=K[G]$ is graded simple with respect to its natural $G$-gradation. However, $R$ is not simple since, for any non-identity $g \in G$, the ideal $R(1-g) R$ is non-trivial.

Remark 9. By assuming that a $G$-graded ring $R$ is graded simple, one often imposes restrictions on the semigroup $G$. In fact, if we suppose that the following rather mild conditions are satisfied

(i) for each non-zero $g \in G$, there is $p, q \in G$ such that $R_{p} R_{g} R_{q}$ is non-zero, and

(ii) there is a non-zero idempotent $e$ in $G$,

then $G$ is an inverse semigroup. In fact, take $g, h \in G$ to be non-zero. By (i), we get that $R R_{g} R$ is a non-zero graded ideal of $R$. Then, by graded simplicity of $R$, we get that $R_{h} \subseteq R R_{g} R$. By (i) again, we get that $R_{h}$ is non-zero. Hence, there are $p, q \in G$ such that $R_{p} R_{g} R_{q}$ is non-zero and $p g q=h$. In other words, we get that $h \in G g G$ for all non-zero $g, h \in G$. Thus, $G$ is simple. This, in combination with the existence of the non-zero idempotent $e$, implies that $G$ is an inverse semigroup (see Theorem 3 in [29]).

Remark 10. Suppose that $G$ is a small category, i.e. with the property that its morphisms form a set. Let the domain and codomain of a morphism $g$ in $G$ be denoted by $d(g)$ and $c(g)$ respectively. Note that every category $G$ can be viewed as a semigroup if we adjoin 
a zero element $\theta$ with the property that $g \theta=\theta g=\theta$, for $g \in G$, and $g h=\theta$ whenever $g h$ is undefined in the category, i.e. when $d(g) \neq c(h)$. As a consequence, category graded rings (in the sense of [28]) can be viewed as semigroup graded rings. Hence, Theorem 2 is applicable in this situation as well. In particular, note that if $G$ is a groupoid, i.e. a category in which all morphisms are invertible, then the semigroup defined by $G$ (as above) is cancellative at all identity morphisms of $G$.

\section{Applications to Partial Skew Group Rings}

In this section, we apply Theorem 2 to partial skew group rings. We generalize a recent result by D. Gonçalves [18] to partial skew group rings by hypercentral groups over rings with local units (see Theorem 17). At the end of this section, we also point out how E. Jespers' result immediately implies a generalization of a simplicity result, recently obtained by A. Baraviera, W. Cortes and M. Soares, for crossed products by twisted partial actions (see Remark [18). First we recall the definition of a partial skew group ring.

Definition 11. Let $G$ be a group with neutral element $e$ and let $A$ be a ring. A partial action $\alpha$ of $G$ on $A$ is a collection of ideals $\left\{D_{g}\right\}_{g \in G}$ of $A$ and a collection of ring isomorphisms $\alpha_{g}: D_{g^{-1}} \rightarrow D_{g}$ such that for all $g, h \in G$ and every $x \in D_{h^{-1}} \cap D_{(g h)^{-1}}$, the following three relations hold:

$$
\text { (i) } \alpha_{e}=\mathrm{id}_{A} ; \quad \text { (ii) } \alpha_{g}\left(D_{g^{-1}} \cap D_{h}\right)=D_{g} \cap D_{g h} ; \quad \text { (iii) } \alpha_{g}\left(\alpha_{h}(x)\right)=\alpha_{g h}(x) .
$$

The partial skew group ring $A \star_{\alpha} G$, associated with the partial action above, is defined as the set of all finite formal sums $\sum_{g \in G} a_{g} \delta_{g}$, where for each $g \in G, a_{g} \in D_{g}$ and $\delta_{g}$ is a symbol. Addition is defined in the obvious way and multiplication is defined as the linear extension of the rule $\left(a_{g} \delta_{g}\right)\left(b_{h} \delta_{h}\right)=\alpha_{g}\left(\alpha_{g^{-1}}\left(a_{g}\right) b_{h}\right) \delta_{g h}$ for $g, h \in G, a_{g} \in D_{g}$ and $b_{h} \in D_{h}$. It is easy to check that if we put $\left(A \star_{\alpha} G\right)_{g}=D_{g} \delta_{g}$, for $g \in G$, then this defines a gradation on the ring $A \star_{\alpha} G$. Clearly, each classical skew group ring (see e.g. [15) is a partial skew group ring where $D_{g}=A$ for all $g \in G$.

Definition 12. Recall from [3] that a ring $R$ has local units if there exists a set $E$ of idempotents in $R$ such that, for every finite subset $X$ of $R$, there exists an $f \in E$ such that $X \subseteq f R f$. From this it follows that $x=f x=x f$ holds for each $x \in X$. In that case, we will refer to $E$ as a set of local units for $R$ and to $f$ as a local unit for the subset $X$.

Remark 13. Rings with local units occur widely in mathematics, often in algebra (e.g. von Neumann regular rings [3, Example 1] and Leavitt path algebras [2, Lemma 1.6]), functional analysis (e.g. algebras of complex-valued functions with compact support) and category theory (e.g. [19, Remark 1] or [16]).

Remark 14. A partial skew group ring $A \star_{\alpha} G$ need not in general be associative (see [13, Example 3.5]). However, if each $D_{g}$, for $g \in G$, has local units, then, in particular, each $D_{g}$, for $g \in G$, is an idempotent ring, i.e. $D_{g}^{2}=D_{g}$, which by [13, Corollary 3.2], ensures that $A \star_{\alpha} G$ is associative. In that case, the set $E \delta_{e}=\left\{f \delta_{e} \mid f \in E\right\}$ is a set of local units for $A \star_{\alpha} G$, if $E$ is a set of local units for $A$. 
Definition 15. If $A \star_{\alpha} G$ is a partial skew group ring, then an ideal $I$ of $A$ is said to be $G$-invariant if $\alpha_{g}\left(I \cap D_{g^{-1}}\right) \subseteq I$ holds for each $g \in G$. If $A$ and $\{0\}$ are the only $G$-invariant ideals of $A$, then $A$ is said to be $G$-simple.

Lemma 16. If $\alpha$ is a partial action of a group $G$ on a ring $A$ such that for each $g \in G$, the ring $D_{g}$ has local units, then $A \star_{\alpha} G$ is graded simple if and only if $A$ is $G$-simple.

Proof. We begin by showing the "only if" statement. Suppose that $A \star_{\alpha} G$ is graded simple. Let $I$ be a non-zero $G$-invariant ideal of $A$. Define $I \star_{\alpha} G$ to be the set of all finite sums of the form $\sum_{g \in G} a_{g} \delta_{g}$, where $a_{g} \in I \cap D_{g}$, for $g \in G$. Note that $I \star_{\alpha} G$ is a non-zero two-sided graded ideal of $A \star_{\alpha} G$. Hence, $I \star_{\alpha} G=A \star_{\alpha} G$. In particular, $A \delta_{e} \subseteq I \star_{\alpha} G$ which shows that $I \subseteq A \subseteq I$. We conclude that $I=A$. Thus, $A$ is $G$-simple.

Now we show the "if" statement. Suppose that $A$ is $G$-simple. Let $J$ be a non-zero graded ideal of $A \star_{\alpha} G$. We claim that $J_{e}=J \cap A$ is a non-zero $G$-invariant ideal of $A$. If we assume that the claim holds, then $A=J_{e}=A \cap J \subseteq J$ from which it follows that $J=A \star_{\alpha} G$. Now we show the claim. First we show that $J_{e}$ is non-zero. Since $J$ is non-zero, there is $g \in G$ and a non-zero $a_{g} \in D_{g}$ with $a_{g} \delta_{g} \in J$. Let $b_{g^{-1}} \in D_{g^{-1}}$ be a local unit for $\alpha_{g^{-1}}\left(a_{g}\right)$. Then $J \ni a_{g} \delta_{g} b_{g^{-1}} \delta_{g^{-1}}=\alpha_{g}\left(\alpha_{g^{-1}}\left(a_{g}\right) b_{g^{-1}}\right) \delta_{e}=\alpha_{g}\left(\alpha_{g^{-1}}\left(a_{g}\right)\right) \delta_{e}=a_{g} \delta_{e}$ which is non-zero. Now we show that $J_{e}$ is $G$-invariant. Take $g \in G$ and $a \in J_{e} \cap D_{g^{-1}}$. Let $c_{g} \in D_{g}$ be such that $\alpha_{g^{-1}}\left(c_{g}\right)$ is a local unit for $a$. Then $\alpha_{g}(a) u_{e}=\alpha_{g}\left(\alpha_{g^{-1}}\left(c_{g}\right) a\right) u_{e}=c_{g} \delta_{g} a \delta_{g^{-1}} \in J$.

The following result generalizes the recent result by D. Gonçalves [18, Theorem 2.5] from the case when $G$ is abelian to the case when $G$ is a hypercentral group. Moreover, our result shows that it is enough to consider the center of one corner.

Theorem 17. Suppose that $\alpha$ is a partial action of a hypercentral group $G$ on a ring $A$ such that for each $g \in G$, the ring $D_{g}$ has local units. Let $E$ denote a set of local units for A. The following three assertions are equivalent:

(i) $A \star_{\alpha} G$ is a simple ring;

(ii) $A$ is $G$-simple and the center of the corner subring $f \delta_{e}\left(A \star_{\alpha} G\right) f \delta_{e}$ is a field, for some non-zero $f \in E$;

(iii) $A$ is $G$-simple and the center of the corner subring $f \delta_{e}\left(A \star_{\alpha} G\right) f \delta_{e}$ is a field, for each non-zero $f \in E$.

Proof. This follows immediately from Lemma [16] and Theorem 2.

Remark 18. Analogously to the way in which Lemma 16 is proven, one can show, under some mild assumptions, that the crossed product by a twisted partial action [12], denoted by $A \star_{\alpha}^{w} G$, is graded simple if and only if $A$ is $G$-simple. Thereby, [4, Theorem 2.25] which was recently observed by A. Baraviera, W. Cortes and M. Soares, can immediately be retrieved as a corollary to [21, Theorem 5]. In fact, by E. Jespers' result (Theorem 11), we note that [4, Theorem 2.25] holds for $A \star_{\alpha}^{w} G$ even when $G$ is a hypercentral (not necessarily abelian) group. 


\section{ACKNOWLEDGEMENTS}

The authors are greatful to an anonymous reviewer for providing insightful comments and suggestions on previous versions of this manuscript. The second author was partially supported by The Swedish Research Council (repatriation grant no. 2012-6113).

\section{REFERENCES}

[1] G. Abrams and C. Menini, Rings of endomorphisms of semigroup-graded modules, Rocky Mountain J. Math. 26 (1996), no. 2, 375-406.

[2] G. Abrams and G. A. Pino, The Leavitt path algebra of a graph, J. Algebra 293 (2005), 319-334.

[3] P. N. Ánh and L. Márki, Morita Equivalence for Rings without Identity, Tsukuba J. Math. 11 (1987), no. 1, 1-16.

[4] A. Baraviera, W. Cortes and M. Soares, Simplicity of Partial Crossed Products, arXiv:1306.5468 [math. RA]

[5] A. D. Bell, Localization and ideal theory in Noetherian strongly group-graded rings, J. Algebra 105 (1987), no. 1, 76-115.

[6] A. D. Bell, S. S. Stalder and M. L. Teply, Prime ideals and radicals in semigroup-graded rings, Proc. Edinburgh Math. Soc. 39 (1996), 1-25.

[7] A. D. Chanyshev, Nilpotency of graded associative algebras, Matem. Sbornik 181 (1990), no. 11, 1573-1579.

[8] H. L. Chick and B. J. Gardner, The preservation of some ring properties by semilattice sums, Comm. Algebra 15 (1987), 1017-1038.

[9] M. V. Clase, E. Jespers and A. del Rio, Semigroup graded rings with finite supports, Glasgow Math. J. 38 (1996), no. 1, 11-18.

[10] K. Crow, Simple Regular Skew Group Rings, J. Algebra Appl. 4 (2005), no. 2, $127-137$.

[11] S. Dascalescu, A. V. Kelarev and L. van Wyk, Semigroup Gradings of Full Matrix Rings, Comm. Algebra 29 (2001), no. 11, 5023-5031.

[12] M. Dokuchaev, R. Exel and J. J. Simón, Crossed products by twisted partial actions and graded algebras, J. Algebra 320 (2008), no. 8, 3278-3310.

[13] M. Dokuchaev and R. Exel, Associativity of crossed products by partial actions, enveloping actions and partial representations. Trans. Amer. Math. Soc. 357 (2005), no. 5, 1931-1952.

[14] R. Exel, Circle actions on $C^{*}$-algebras, partial automorphisms, and a generalized Pimsner-Voiculescu exact sequence, J. Funct. Anal. 122 (1994), no. 2, 361-401.

[15] J. W. Fisher and S. Montgomery, Semiprime skew group rings, J. Algebra 52 (1978), no. 1, 241-247.

[16] K. R. Fuller, Rings with Finiteness Coditions and Their Categories of Functors, J. Algebra 55 (1978), 94-105.

[17] B. J. Gardner, Radicals of supplementary semilattice sums of rings, Pacific J. Math. 58 (1975), 387392.

[18] D. Gonçalves, Simplicity of partial skew group rings of abelian groups, Canad. Math. Bull. 57 (2014), no. 3, 511-519.

[19] M. Harada, Perfect Categories I, Osaka J. Math., vol. 10 (1973), 329-367.

[20] V. V. Ignatov, On the semihereditariness of semilattice sums of rings, Abelian Groups and Modules, Tomsk, (1982) 110-116.

[21] E. Jespers, Simple abelian-group graded rings, Boll. Un. Mat. Ital. A (7) 3 (1989), no. 1, $103-106$.

[22] E. Jespers, Simple Graded Rings, Comm. Algebra 21(1993), no. 7, 2437-2444.

[23] G. Karpilovsky, The Jacobson radical of monoid-graded algebras, Tsukuba J. Math. 16 (1992), $19-52$.

[24] A. V. Kelarev, Applications of epigroups to graded ring theory, Semigroup Forum 50 (1995), 327-350.

[25] A. V. Kelarev, Semisimple Rings Graded by Inverse Semigroups, J. Algebra 205 (1998), 451-459.

[26] A. V. Kelarev, Ring Constructions and Applications, World Scientific (2002). 
[27] J. Krempa and J. Okninski, Group rings which are Jacobson rings, Arch. Math. (Basel) 44 (1985), no. $1,20-25$.

[28] P. Lundström, The category of groupoid graded modules, Colloq. Math. 100 (2004), no. 2, 195-211.

[29] R. McFadden and R. Schneider, Completely Simple and Inverse Semigroups, Proc. Cambridge Philos. Soc. 57 (1961), 234-236.

[30] W. D. Munn, Inverse semigroup rings, Topics in algebra, Warsaw, Banach Center Publ., 26 (1988), Part 1, Warsaw, 65-73.

[31] P. Nystedt and J. Öinert, Simple skew category algebras associated with minimal partially defined dynamical systems, Discrete Contin. Dyn. Syst. 33 (2013), no. 9, 4157-4171.

[32] P. Nystedt and J. Öinert, Simple rings and degree maps, J. Algebra 401 (2014), 201-219.

[33] J. Öinert, Simplicity of skew group rings of abelian groups, Comm. Algebra 42 (2014), no. 2, 831-841.

[34] J. S. Ponizovskii, Semigroup rings, Semigroup Forum 36 (1987), 1-46.

[35] E. R. Puczylowski, A note on graded algebras, Proc. Amer. Math. Soc. 113 (1) (1991), 1-3.

[36] D. J. S. Robinson, Finiteness Conditions and Generalized Soluble Groups, Part I, Ergebnisse der Mathematik und ihrer Grenzgebiete, No. 62, Springer-Verlag, Berlin, 1972.

[37] P. Wauters and E. Jespers, When is a semigroup ring of a commutative semigroup local or semilocal?, J. Algebra 108 (1987), no. 1, 188-194. 